\title{
Indices of 1-forms on an isolated complete intersection singularity
}

\author{
W.Ebeling and S.M.Gusein-Zade *
}

\section{Introduction (1-forms versus vector fields)}

There is a number of papers devoted to the definition and the calculation of the index of an analytic vector field on a real analytic variety with an isolated singular point ([ASV], EGZ, [GMM1, [GMM2]). In |GSV] there was defined a notion of the index of a holomorphic vector field on an isolated complete intersection singularity (icis). One can say that the reason for inventing this notion is the hope that it can be used for calculation of the index of a vector field on a real icis.

We offer a different approach. Instead of considering vector fields on a variety we consider 1-forms. One can define the notions of the index of a real 1 -form on a germ of a real analytic variety with an isolated singular point and of a holomorphic 1-form on a (complex) icis. (For short sometimes we shall refer to these two settings as to the real and the complex indices respectively.) To a vector field on a real variety $(V, 0) \subset\left(\mathbb{R}^{n}, 0\right)$ (or on a complex analytic variety $\left.(V, 0) \subset\left(\mathbb{C}^{n}, 0\right)\right)$ one can associate a 1 -form on it (dependent on the choice of coordinates in $\left(\mathbb{R}^{n}, 0\right)$ or in $\left.\left(\mathbb{C}^{n}, 0\right)\right)$. If the vector field has an algebraically isolated singular point on $(V, 0)$ (in the complex setting this simply means isolatedness), then, for a generic choice of coordinates, the corresponding 1-form has an algebraically isolated singular point as well. This correspondence does not work in the other direction. Moreover the index of a vector field on a real analytic variety with an isolated singular point coincides with that of the corresponding 1-form. The notion of the index of a holomorphic 1-form on an icis is somewhat more natural than that of a holomorphic vector field: in some sense it is "more complex analytic" (does not use the complex conjugation for the definition) and "more geometric" (uses only objects of the same tensor type). Moreover the index of an isolated singular point of a holomorphic 1-form on a complex icis can be described as the dimension of an appropriate algebra. Finally, the real index of a 1-form on a real icis (with an algebraically isolated

*Partially supported by the DFG-programme "Global methods in complex geometry", grants RFBR-, INTAS-00-259. 
singular point) plus-minus the Euler characteristic of a (real) smoothing of the icis can be expressed in terms of the signature of a (nondegenerate) quadratic form on a space, the dimension of which is equal to the (complex) index of the corresponding complexification.

The idea to consider indices of 1-forms instead of indices of vector fields (in some situations) was first formulated by V.I.Arnold ([A]). We are thankful to E.Looijenga, M.Merle and J.Briançon for useful discussions. In particular the comments of E.Looijenga helped us to prove Theorem 1 .

\section{Indices of real 1-forms on singular varieties}

A manifold with isolated singularities is a topological space $M$ which has the structure of a smooth (say, $C^{\infty}$-) manifold outside of a discrete set $S$ (the set of singular points of $M$ ). A diffeomorphism between two such manifolds is a homeomorphism which sends the set of singular points onto the set of singular points and is a diffeomorphism outside of them. We say that $M$ has a conelike singularity at a (singular) point $P \in S$ if there exists a neighbourhood of the point $P$ diffeomorphic to the cone $C W_{P}=\left(I \times W_{P}\right) /\left(\{0\} \times W_{P}\right)(I=$ $[0,1])$ over a smooth manifold $W_{P}\left(W_{P}\right.$ is called the link of the point $\left.P\right)$. In what follows we assume all manifolds to have only cone-like singularities. In [EGZ we discussed the notion of a vector field on a manifold with isolated singularities and the notion of the index of its singular point. Here we adapt the corresponding definitions to the case of 1-forms. A (smooth or continuous) 1 -form on a manifold $M$ with isolated singularities is a (smooth or continuous) 1 -form on the set $M \backslash S$ of regular points of $M$. The set of singular points $S_{\omega}$ of a 1-form $\omega$ on a (singular) manifold $M$ is the union of the set of usual singular points of $\omega$ on $M \backslash S$ (i.e., points at which $\omega$ tends to zero) and of the set $S$ of singular points of $M$ itself.

For an isolated usual singular point $P$ of a 1-form $\omega$ there is defined its index $\operatorname{ind}_{P} \omega$ (the degree of the map $\omega /\|\omega\|: \partial B \rightarrow S^{n-1}$ of the boundary of a small ball $B$ centred at the point $P$ in a coordinate neighbourhood of $P$ into the unit sphere in the dual space; $n=\operatorname{dim} M$ ). If the manifold $M$ is closed (i.e., compact, without boundary), has no singularities $(S=\emptyset)$, and the 1-form $\omega$ on $M$ has only isolated singularities, then

$$
\sum_{P \in S_{\omega}} \operatorname{ind}_{P} \omega=\chi(M)
$$

$(\chi(M)$ is the Euler characteristic of $M)$.

Let $(M, P)$ be a cone-like singularity (i.e., a germ of a manifold with such a singular point) and let $\omega$ be a 1-form defined on an open neighbourhood $U$ of the point $P$. Suppose that $\omega$ has no singular points on $U \backslash\{P\}$. Let $V$ be a closed cone-like neighbourhood of $P$ in $U\left(V \cong C W_{P}, V \subset U\right)$. On the cone $C W_{P}=\left(I \times W_{P}\right) /\left(\{0\} \times W_{P}\right)(I=[0,1])$ there is defined a natural 1-form $d t$ 
( $t$ is the coordinate on $I$ ). Let $\omega_{\text {rad }}$ be the corresponding 1-form on $V$. Let $\widetilde{\omega}$ be a smooth 1-form on $U$ which coincides with $\omega$ near the boundary $\partial U$ of the neighbourhood $U$ and with $\omega_{\text {rad }}$ on $V$ and has only isolated singular points.

Definition: The index $\operatorname{ind}_{P} \omega$ of the 1 -form $\omega$ at the point $P$ is equal to

$$
1+\sum_{Q \in S_{\omega} \backslash\{P\}} \operatorname{ind}_{Q} \widetilde{\omega}
$$

(the sum is over all singular points $Q$ of the 1 -form $\widetilde{\omega}$ except $P$ itself).

For a cone-like singularity at a point $P \in S$, the link $W_{P}$ and thus the cone structure of a neighbourhood are, generally speaking, not well-defined (cones over different manifolds may be locally diffeomorphic). However it is not difficult to show that the index ind $P \omega$ does not depend on the choice of the cone structure on a neighbourhood and on the choice of the 1-form $\widetilde{\omega}$.

Proposition 1 For a 1-form $\omega$ with isolated singular points on a closed manifold $M$ with isolated singularities, the relation (11) holds.

Definition: One says that a singular point $P$ of a manifold $M$ (locally diffeomorphic to the cone $C W_{P}$ over a manifold $W_{P}$ ) is smoothable if $W_{P}$ is the boundary of a smooth compact manifold $\widetilde{V}_{P}$.

In what follows we shall call $\widetilde{V}_{P}$ a smoothing of $(V, P)$. The class of smoothable singularities includes, in particular, the class of (real) isolated complete intersection singularities. For such a singularity, there is a distinguished conelike structure on its neighbourhood.

Let $(M, P)$ be a smoothable singularity (i.e., a germ of a manifold with such a singular point) and let $\omega$ be a 1-form on $(M, P)$ with an isolated singular point at $P$. Let $V=C W_{P}$ be a closed cone-like neighbourhood of the point $P ; \omega$ is supposed to have no singular points on $V \backslash\{P\}$. Let the link $W_{P}$ of the point $P$ be the boundary of a compact manifold $\widetilde{V}_{P}$. Identifying $\partial \widetilde{V}_{P}=W_{P}$ with $W_{P} \times\{1 / 2\}$ and using a smoothing one can consider the union $\widetilde{V}_{P} \cup_{W_{P}}\left(W_{P} \times\right.$ $[1 / 2,1])$ of $\widetilde{V}_{P}$ and $W_{P} \times[1 / 2,1] \subset C W_{P}$ glued along the common boundary as a smooth manifold (with the boundary $W_{P} \times\{1\}$ ). The restriction of the 1-form $\omega$ to $W_{P} \times[1 / 2,1] \subset C W_{P}=V$ can be extended to a smooth 1-form $\widetilde{\omega}$ on $\widetilde{V}_{P} \cup_{W_{P}}\left(W_{P} \times[1 / 2,1]\right)$ with isolated singular points.

Proposition 2 The index $\operatorname{ind}_{P} \omega$ of the 1-form $\omega$ at the point $P$ is equal to

$$
\sum_{Q \in S_{\tilde{\omega}}} \operatorname{ind}_{Q} \widetilde{\omega}-\chi\left(\widetilde{V}_{P}\right)+1
$$

(the sum is over all singular points of $\widetilde{\omega}$ on $\widetilde{V}_{P}$ ). 
Let $(V, 0) \subset\left(\mathbb{R}^{n}, 0\right)$ be a real $(n-k)$-dimensional (from the topological point of view as well) variety with an isolated singularity at the origin. Let $X$ be an analytic vector field on $(V, 0)$, that is the restriction of an analytic vector field $\sum X_{i} \frac{\partial}{\partial x_{i}}$ (which we shall denote by $X$ as well) defined on a neighbourhood of the origin in $\mathbb{R}^{n}$ and tangent to the variety $V$ (outside of the origin). Suppose that the origin is an isolated singular point of the vector field $X$ on $V$, i.e., $X$ has no zeros on $V$ outside of the origin (in a neighbourhood of it). In this situation the index $\operatorname{ind}_{0} X$ of the vector field $X$ at the origin is defined (see [EGZ]). Let $\omega$ be the 1 -form $\sum X_{i} d x_{i}$. The 1-form $\omega$ on $V$ has an isolated singular point at the origin as well. Moreover $\operatorname{ind}_{0} \omega=\operatorname{ind}_{0} X$. Thus in this case the problem of calculating the index of a vector field can be reduced to the problem of calculating the index of a 1 -form. This correspondence does not work in the other direction: for a 1 -form $\omega=\sum A_{i} d x_{i}$, the vector field $\sum A_{i} \frac{\partial}{\partial x_{i}}$, generally speaking, is not tangent to the variety $V$.

Remark. The described 1-form $\omega$ corresponding to a vector field $X$ on $V$ depends on the choice of the coordinates on $\left(\mathbb{R}^{n}, 0\right)$.

One can hope to get an algebraic formula for the index of a vector field or of a 1-form on a singular variety $(V, 0) \subset\left(\mathbb{R}^{n}, 0\right)$ with an isolated singular point at the origin in the spirit of the Eisenbud-Levine-Khimshiashvili one ( $\mathbb{E L}]$, [Kh]) only if the origin is an algebraically isolated singular point of the vector field or of the 1-form. This means that the complexification of the vector field (or of the 1 -form) on the complexification $\left(V_{\mathbb{C}}, 0\right) \subset\left(\mathbb{C}^{n}, 0\right)$ of the variety $(V, 0)$ has no zeros on $V_{\mathbb{C}}$ outside of the origin (in a neighbourhood of it; one supposes that $V$ has an algebraically isolated singular point at the origin itself, i.e., $V_{\mathbb{C}}$ has an isolated singular point at the origin). Suppose that the vector field $X=\sum X_{i} \frac{\partial}{\partial x_{i}}$ has an algebraically isolated singular point at the origin. In this case the 1-form $\omega=\sum X_{i} d x_{i}$ on $V_{\mathbb{C}}$ may have a nonisolated singular point at it. For instance, this takes place for $V=\left\{(x, y, z) \in \mathbb{R}^{3}: x^{2}+y^{2}-z^{2}=0\right\}$, $X=x \frac{\partial}{\partial x}+y \frac{\partial}{\partial y}+z \frac{\partial}{\partial z}$. In such case one hardly can hope to have an algebraic formula for the index ind $0 \omega$ of the 1-form $\omega$. The following statement helps to avoid this difficulty.

Lemma 1 Let $X=\sum X_{i} \frac{\partial}{\partial x_{i}}$ be a vector field tangent to $V$ which has an algebraically isolated (on $V$ ) singular point at the origin. Then after a possible analytic change of coordinates in $\left(\mathbb{R}^{n}, 0\right)$ (in fact after a generic one), the associated 1-form $\omega=\sum X_{i} d x_{i}$ on $V$ has an algebraically isolated singular point at the origin as well.

Proof. Consider the subset $\Xi$ of the space $J^{1}\left(V_{\mathbb{C}} \backslash\{0\}, \mathbb{C}^{n}\right)$ of 1-jets of maps from $V_{\mathbb{C}} \backslash\{0\}$ to $\mathbb{C}^{n}$ which consists of jets $(F(x), d F(x))\left(x \in V_{\mathbb{C}} \backslash\{0\}, F(x) \in\right.$ $\left.\mathbb{C}^{n}, d F(x): T_{x} V_{\mathbb{C}} \rightarrow T_{F(x)} \mathbb{C}^{n}=\mathbb{C}^{n}\right)$ such that $d F(x)(X(x))$ is orthogonal to $\operatorname{Im} d F(x)$ in the sense of the quadratic form $\sum_{i=1}^{n} z_{i}^{2}$ on $\mathbb{C}^{n}$. $\Xi$ is a submanifold of $J^{1}\left(V \backslash\{0\}, \mathbb{C}^{n}\right)$ of codimension $n-k=\operatorname{dim} V_{\mathbb{C}}$. For an immersion $F$ : 
$V_{\mathbb{C}} \backslash\{0\} \rightarrow \mathbb{C}^{n}$ the intersection points of the image of the jet extension $j^{1} F$ of the map $F$ with $\Xi$ are just those points where the 1-form corresponding to the vector field $F_{*} X$ vanishes on $T_{F(x)} F\left(V_{\mathbb{C}} \backslash\{0\}\right)$. The Strong Transversality Theorem implies that, for a generic change of coordinates, the image of $V_{\mathbb{C}} \backslash\{0\}$ intersects $\Sigma$ (transversally) at isolated points. Now the fact that after the change of coordinates the 1-form corresponding to the vector field under consideration has an algebraically isolated singular point at the origin follows from the Curve Selection Lemma.

\section{Index of a holomorphic 1-form on an icis}

Let $f=\left(f_{1}, \ldots, f_{k}\right):\left(\mathbb{C}^{n}, 0\right) \rightarrow\left(\mathbb{C}^{k}, 0\right)$ be an analytic map which defines an $(n-k)$-dimensional icis $V=f^{-1}(0) \subset\left(\mathbb{C}^{n}, 0\right)\left(f_{i}:\left(\mathbb{C}^{n}, 0\right) \rightarrow(\mathbb{C}, 0)\right)$.

For a germ of a holomorphic vector field $X$ which is tangent to $V$ and has an isolated singular point on $V$ at the origin, an index is defined in [GSV] and [SS]. We recall the definition of this index. Let $U$ be a neighbourhood of the origin in $\mathbb{C}^{n}$ where all the functions $f_{i}(i=1, \ldots, k)$ and the vector field $X$ are defined. Let $S_{\delta} \subset U$ be a sufficiently small sphere around the origin which intersects $V$ transversally. Let $K=V \cap S_{\delta}$ be the link of the icis $(V, 0)$. Define grad $f_{i}$ by

$$
\operatorname{grad} f_{i}=\left(\overline{\frac{\partial f_{i}}{\partial x_{1}}}, \ldots, \overline{\frac{\partial f_{i}}{\partial x_{n}}}\right) .
$$

One has a map

$$
\left(X, \operatorname{grad} f_{1}, \ldots, \operatorname{grad} f_{k}\right): K \rightarrow W_{k+1}\left(\mathbb{C}^{n}\right),
$$

where $W_{k+1}\left(\mathbb{C}^{n}\right)$ is the Stiefel manifold of $(k+1)$-frames in $\mathbb{C}^{n}$. It is well-known that $W_{k+1}\left(\mathbb{C}^{n}\right)$ is $(2(n-k)-2)$-connected and that $H_{2(n-k)-1}\left(W_{k+1}\left(\mathbb{C}^{n}\right)\right) \cong$ $\pi_{2(n-k)-1}\left(W_{k+1}\left(\mathbb{C}^{n}\right)\right) \cong \mathbb{Z}$ (see, e.g., $[\mathbb{H}]$ ). On the other hand, $K$ is a smooth manifold of dimension $2(n-k)-1$ with the natural orientation as the boundary of the complex manifold $V \backslash\{0\}$. Therefore a map from $K$ to $W_{k+1}\left(\mathbb{C}^{n}\right)$ has a degree. The index of the vector field $X$ at the origin is defined to be the degree of the above map.

Remark. Pay attention that one uses the complex conjugation for this definition and the components of the discussed map are of different tensor nature. Whereas $X$ is a vector field, grad $f_{i}$ is more similar to a covector.

Now we adapt the definition of the index of a holomorphic vector field to the case of a holomorphic 1-form on $V$. Let $\omega=\sum A_{i} d x_{i}\left(A_{i}=A_{i}(x)\right)$ be a germ of a holomorphic 1-form on $\left(\mathbb{C}^{n}, 0\right)$ which as a 1-form on $V$ has (at most) an isolated singular point at the origin (thus it does not vanish on the tangent space $T_{P} V$ to the variety $V$ at all points $P$ from a punctured neighbourhood of 
the origin in $V)$. The 1 -forms $\omega, d f_{1}, \ldots, d f_{k}$ are linearly independent for all $P \in K$. Thus one has a map

$$
\left(\omega, d f_{1}, \ldots, d f_{k}\right): K \rightarrow W_{k+1}\left(\mathbb{C}^{n}\right) .
$$

Definition: We define the index of the 1 -form $\omega$ at 0 , $\operatorname{ind}_{\mathbb{C}, 0} \omega$, to be the degree of the map

$$
\left(\omega, d f_{1}, \ldots, d f_{k}\right): K \rightarrow W_{k+1}\left(\mathbb{C}^{n}\right)
$$

(here $W_{k+1}\left(\mathbb{C}^{n}\right)$ is the manifold of $(k+1)$-frames in the dual $\left.\mathbb{C}^{n}\right)$.

Remark. The Stiefel manifold $W_{k+1}\left(\mathbb{C}^{n}\right)$ of $(k+1)$-frames in $\mathbb{C}^{n}$ is homotopy equivalent to the (Stiefel) manifold $\widetilde{W}_{k+1}\left(\mathbb{C}^{n}\right)$ of orthonormal (with respect to the Hermitian scalar product $\left.\sum x_{i} \overline{y_{j}}\right)(k+1)$-frames in $\mathbb{C}^{n}$. The homotopy equivalence is defined by the Gram-Schmidt process. Thus in the definition one can substitute $W_{k+1}\left(\mathbb{C}^{n}\right)$ by $\widetilde{W}_{k+1}\left(\mathbb{C}^{n}\right)$ and the map

$$
\left(\omega, d f_{1}, \ldots, d f_{k}\right): K \rightarrow W_{k+1}\left(\mathbb{C}^{n}\right)
$$

by the corresponding map

$$
\left(\omega, d f_{1}, \ldots, d f_{k}\right)^{\sim}: K \rightarrow \widetilde{W}_{k+1}\left(\mathbb{C}^{n}\right) .
$$

However $\widetilde{W}_{k+1}\left(\mathbb{C}^{n}\right)$ is not a complex analytic manifold and thus the map

$$
\left(\omega, d f_{1}, \ldots, d f_{k}\right)^{\sim}: U \backslash\{0\} \rightarrow \widetilde{W}_{k+1}\left(\mathbb{C}^{n}\right)
$$

of a punctured neighbourhood $U \backslash\{0\}$ of the origin in $V$ is not complex analytic (in contrast with the map $\left.\left(\omega, d f_{1}, \ldots, d f_{k}\right): U \backslash\{0\} \rightarrow W_{k+1}\left(\mathbb{C}^{n}\right)\right)$. We prefer to give a "more complex analytic" definition, using sometimes the map to $\widetilde{W}_{k+1}\left(\mathbb{C}^{n}\right)$ for calculations.

Example. Let $n=2, k=1, f_{1}(x, y)=x^{2}+y^{3}$ and consider the 1-form

$$
\omega=3 y^{2} d x-2 x d y .
$$

The form $\omega$ on $V=\left\{f_{1}=0\right\}$ has an isolated zero at the origin. One can easily compute that the degree of the map

$$
\left(\omega, d f_{1}\right)^{\sim}: K \rightarrow \widetilde{W}_{2}\left(\mathbb{C}^{2}\right) \cong U(2)
$$

is 6. Therefore ind $\mathbb{C}, 0_{\mathbb{1}} \omega=6$ (see also Proposition 3). This 1-form corresponds (in the described sense) to the vector field

$$
X=3 y^{2} \frac{\partial}{\partial x}-2 x \frac{\partial}{\partial y} .
$$


This vector field (by chance) is tangent to the hypersurface $V=\left\{f_{1}=0\right\}$ and has an isolated singular point at the origin on it, but its index is 0 , since the map

$$
\left(X, \operatorname{grad} f_{1}\right)^{\sim}: K \rightarrow \widetilde{W}_{2}\left(\mathbb{C}^{2}\right) \cong U(2)
$$

maps $K$ to $S U(2)$ which is simply connected.

Let $B_{\delta}$ be the ball of radius $\delta$ around the origin in $\mathbb{C}^{n}$ with the boundary $S_{\delta}$. Suppose that the functions $f_{1}, \ldots, f_{k}$ and the 1 -form $\omega$ are defined on a neighbourhood of $B_{\delta}$ and let $\varepsilon=\left(\varepsilon_{1}, \ldots, \varepsilon_{k}\right) \in \mathbb{C}^{k}$ be small enough (so that the level set $V_{\varepsilon}:=f^{-1}(\varepsilon) \cap B_{\delta}$ is transversal to the sphere $\left.S_{\delta}\right)$ and such that this level set $V_{\varepsilon}$ is nonsingular.

Definition: Let $M$ be a complex $m$-dimensional manifold and let $\eta$ be a holomorphic 1-form on $M$. A zero $P \in M$ of the 1-form $\eta$ is called nondegenerate if in local coordinates $y_{1}, \ldots, y_{m}$ on $M$ around $P$ in which the 1 -form $\eta$ is written as $\eta=C_{1} d y_{1}+\cdots C_{m} d y_{m}$, the Hessian of $\eta$ at the point $P$, i.e., the determinant of the matrix

$$
\left(\frac{\partial C_{i}}{\partial y_{j}}(P)\right)_{i, j=1, \ldots, m},
$$

is nonzero.

There exists a perturbation $\widetilde{\omega}$ of the 1 -form $\omega$ which has only nondegenerate zeros on $V_{\varepsilon}$. (In fact a generic perturbation of $\omega$ and, in particular, a perturbation of the form $\widetilde{\omega}=\omega-\lambda \eta$ for a generic 1 -form $\eta$ on $\mathbb{C}^{n}$ (where $\lambda \neq 0$ is small enough) possesses this property.)

Proposition 3 The index ind $_{\mathbb{C}, 0} \omega$ of the 1-form $\omega$ on the icis $V$ at the origin is equal to the number of zeros of $\omega$ on $V_{\varepsilon}$, counted with multiplicities. It is also equal to the number of zeros of $\widetilde{\omega}$ on $V_{\varepsilon}$ for a small perturbation $\widetilde{\omega}$ of the 1-form $\omega$ with only nondegenerate zeros on $V_{\varepsilon}$.

Proof. Let $P_{1}, \ldots, P_{\nu}$ be the zeros of the form $\omega$ on $V_{\varepsilon}$. In local coordinates $y_{1}$, $\ldots, y_{k}, y_{k+1}, \ldots, y_{n}$ centred at the point $P_{i}$ such that $V_{\varepsilon}=\left\{y_{1}=\ldots=y_{k}=0\right\}$ (one can take $y_{i}=f_{i}-\varepsilon_{i}$ for $1 \leq i \leq k$ ) let $\omega_{\mid V_{\varepsilon}}=C_{k+1} d y_{k+1}+\ldots+C_{n} d y_{n}$. Let $B_{i}$ be a small open ball centred at the point $P_{i}$. The degree of the map $\left(\omega, d f_{1}, \ldots, d f_{k}\right): \partial B_{i} \cap V_{\varepsilon} \rightarrow W_{k+1}\left(\mathbb{C}^{n}\right)$ is equal to the degree of the map $S^{2(n-k)-1} \rightarrow S^{2(n-k)-1}$ given by

$$
x \mapsto\left(C_{k+1}, \ldots, C_{n}\right) /\left\|\left(C_{k+1}, \ldots, C_{n}\right)\right\|
$$

and thus is equal to the multiplicity $\mu_{i}$ of the zero $P_{i}$ (see, e.g., AGV). Now consider the manifold

$$
M:=V_{\varepsilon} \backslash \bigcup_{i} B_{i} .
$$

Since the 1-form $\omega$ has no zeros on $M$, the degree of the mapping

$$
\left(\omega, d f_{1}, \ldots, d f_{k}\right): \partial M \rightarrow W_{k+1}\left(\mathbb{C}^{n}\right)
$$


is equal to zero. This implies

$$
\operatorname{ind}_{\mathbb{C}, 0} \omega=\sum_{i=1}^{\nu} \mu_{i}
$$

If $\widetilde{\omega}$ is a perturbation of the 1-form $\omega$ with only nondegenerate zeros on $V_{\varepsilon}$ then it has just $\mu_{i}$ nondegenerate zeros on $B_{i} \cap V_{\varepsilon}$.

\section{An algebraic formula for the index}

Our aim in this section is to derive the following algebraic formula for the complex index.

Theorem 1 Let $\mathcal{O}_{\mathbb{C}^{n}, 0}$ be the algebra of germs of holomorphic functions at the origin in $\mathbb{C}^{n}$ and let $I \subset \mathcal{O}_{\mathbb{C}^{n}, 0}$ be the ideal generated by $f_{1}, \ldots, f_{k}$ and the $(k+1) \times(k+1)$-minors of the matrix

$$
\left(\begin{array}{ccc}
\frac{\partial f_{1}}{\partial x_{1}} & \cdots & \frac{\partial f_{1}}{\partial x_{n}} \\
\vdots & \ddots & \vdots \\
\frac{\partial f_{k}}{\partial x_{1}} & \cdots & \frac{\partial f_{k}}{\partial x_{n}} \\
A_{1} & \cdots & A_{n}
\end{array}\right)
$$

Then

$$
\operatorname{ind}_{\mathbb{C}, 0} \omega=\operatorname{dim}_{\mathbb{C}} \mathcal{O}_{\mathbb{C}^{n}, 0} / I
$$

Remark. A corresponding result for the case when the 1-form $\omega$ is the differential $d f_{k+1}$ of a function $f_{k+1}$ was first proven by G.-M.Greuel in [G, Lemma 1.9]. G.-M.Greuel has informed us that his arguments in that paper can be adapted to prove Theorem 1 as well.

The proof will rely on a basic fact which we now want to formulate.

Let $F:\left(\mathbb{C}^{n} \times \mathbb{C}^{M}, 0\right) \rightarrow\left(\mathbb{C}^{k} \times \mathbb{C}^{M}, 0\right)$ be a versal deformation of $f:\left(\mathbb{C}^{n}, 0\right) \rightarrow$ $\left(\mathbb{C}^{k}, 0\right)$, let $N:=n+M, K:=k+M$. By the same symbol, $F$, we denote a representative $U \rightarrow \mathbb{C}^{K}$ of this deformation defined in a small open neighbourhood $U \subset \mathbb{C}^{N}$ of the origin. Let $A_{i}=0$ for $i=n+1, \ldots, N$ and let $\eta=\sum_{i=1}^{N} B_{i} d x_{i}$ be a 1-form such that for a regular value $s \in F(U)$ of $F$ and for $\lambda \in W, \lambda \neq 0$, where $W \subset \mathbb{C}$ is a suitable small open neighbourhood of the origin, the form $\omega-\lambda \eta$ has only isolated zeros on $F^{-1}(s)$.

Consider the matrix

$$
\Phi=\left(\begin{array}{ccc}
\frac{\partial F_{1}}{\partial x_{1}} & \cdots & \frac{\partial F_{1}}{\partial x_{N}} \\
\vdots & \ddots & \vdots \\
\frac{\partial F_{K}}{\partial x_{1}} & \cdots & \frac{\partial F_{K}}{\partial x_{N}} \\
A_{1}-\lambda B_{1} & \cdots & A_{N}-\lambda B_{N}
\end{array}\right) .
$$


Let $\mathbb{C}^{N+1}$ be the vector space with coordinates $x_{1}, \ldots, x_{N}, \lambda$. Let $\mathcal{C}(U \times W)$ denote the ideal in $\mathcal{O}_{\mathbb{C}^{N+1}}(U \times W)$ generated by the $(K+1) \times(K+1)$ minors of the matrix $\Phi$. Let $\mathcal{C} \subset \mathcal{O}_{\mathbb{C}^{N+1}}$ denote the corresponding ideal sheaf and let $C \subset U \times W$ be the analytic space defined by $\mathcal{C}$. Then $C$ consists of those points $x \in U \times W$ where $F$ is not a submersion or $\omega-\lambda \eta$ does not have an isolated zero in $x \in F^{-1}(F(x))$. Let $\mathcal{O}_{C}:=\mathcal{O}_{\mathbb{C}^{N+1}} / \mathcal{C}$ be the structure sheaf of $C$.

Let $\widetilde{F}: U \times \mathbb{C} \rightarrow \mathbb{C}^{K+1}$ be the mapping defined by $(x, \lambda) \mapsto(F(x), \lambda)$. Let $\Sigma:=\widetilde{F}(C)$ and let $\pi:=\left.\widetilde{F}\right|_{C}: C \rightarrow \Sigma$. Then $\Sigma$ is an analytic space endowed with the structure sheaf $\mathcal{O}_{\Sigma}:=\mathcal{O}_{\mathbb{C}^{K+1}} / \mathcal{F}_{0}\left(\pi_{*}\left(\mathcal{O}_{C}\right)\right.$ ) (restricted to $\left.\pi(C)\right)$ where $\mathcal{F}_{0}$ denotes the 0 -th Fitting ideal (cf. [4, 4.E]). The mapping $\pi: C \rightarrow \Sigma$ is a finite mapping.

Theorem 1 will follow from the next theorem.

Theorem 2 For every $x \in C, \mathcal{O}_{C, x}$ is a flat $\mathcal{O}_{\Sigma, \pi(x)}$-module.

Proof. The proof follows the same lines as [L, (4.4) and (4.8)].

Let $x \in C$ and $s=\pi(x)$. The minors of the matrix $\Phi$ vanish at a point $x \in U \times W$ if and only if the rank of the matrix $\Phi(x)$ is not maximal. The set of $(N+1) \times(K+1)$ matrices with complex entries with rank $<K+1$ is an affine algebraic variety of codimension $N-K+1$ inside the set of all $(N+1) \times(K+1)$ matrices with complex entries. Hence $C$ has dimension $K$. This implies that $\operatorname{depth}\left(\mathcal{C}_{x} ; \mathcal{O}_{\mathbb{C}^{N+1}, x}\right)=N-K+1$. By [BR, Corollary (2.7)] this implies that the homological dimension hd $\mathcal{C}_{x}$ of $\mathcal{C}_{x}$ is equal to $N-K+1$.

By the formula of Auslander-Buchsbaum (see, e.g., [Ma, p. 114]) we have

$$
\operatorname{depth} \mathcal{O}_{C, x}=N+1-(N-K+1)=K .
$$

Since also $\operatorname{dim} \mathcal{O}_{C, x}=K$, it follows that $\mathcal{O}_{C, x}$ is a Cohen-Macaulay ring.

Since $\left.\widetilde{F}\right|_{C}: C \rightarrow \mathbb{C}^{K+1}$ is a finite mapping, $\mathcal{O}_{C, x}$ is a finite $\mathcal{O}_{\mathbb{C}^{K+1}, s}$-module. Therefore $\mathcal{O}_{C, x}$ is a Cohen-Macaulay module over $\mathcal{O}_{\mathbb{C}^{K+1}, s}$ and

$$
\operatorname{depth}_{\mathcal{O}_{\mathbb{C} K+1, s}} \mathcal{O}_{C, x}=\operatorname{dim} \mathcal{O}_{C, x}=K
$$

Since $\mathcal{O}_{\mathbb{C}^{K+1}, s}$ is an $(K+1)$-dimensional regular ring, it follows from the Auslander-Buchsbaum formula that the homological dimension of $\mathcal{O}_{C, x}$ as an $\mathcal{O}_{\mathbb{C}^{K+1}, s^{-}}$ module is 1 . This means that there is an exact sequence of $\mathcal{O}_{\mathbb{C}^{K+1}, s^{-} \text {modules }}$

$$
0 \rightarrow \mathcal{O}_{\mathbb{C}^{K+1}, s}^{q} \stackrel{\alpha}{\rightarrow} \mathcal{O}_{\mathbb{C}^{K+1}, s}^{p} \rightarrow \mathcal{O}_{C, x} \rightarrow 0 .
$$

Moreover, $q$ must be equal to $p$ and the 0 -th Fitting ideal of $\mathcal{O}_{C, x}$ viewed as an $\mathcal{O}_{\mathbb{C}^{K+1}, s}$-module is generated by the determinant of $\alpha$. Hence $\Sigma$ is a hypersurface and $\mathcal{O}_{\Sigma, s}$ is a Cohen-Macaulay ring, too.

Since $\pi: C \rightarrow \Sigma$ is a finite mapping, $\mathcal{O}_{C, x}$ is also a finite $\mathcal{O}_{\Sigma, s}$-module. But a finitely generated $\mathcal{O}_{\Sigma, s}$-module is flat if and only if it is free (see e.g. Ma, (3.G) 
Proposition]). Therefore it suffices to show that $\mathcal{O}_{C, x}$ is a free $\mathcal{O}_{\Sigma, s}$-module. By the Auslander-Buchsbaum formula we have

$$
\operatorname{hd}_{\mathcal{O}_{\Sigma, s}} \mathcal{O}_{C, x}+\operatorname{depth}_{\mathcal{O}_{\Sigma, s}} \mathcal{O}_{C, x}=\operatorname{depth} \mathcal{O}_{\Sigma, s}
$$

Since depth $\mathcal{O}_{\Sigma, s} \mathcal{O}_{C, x}=\operatorname{depth} \mathcal{O}_{\Sigma, s}=\operatorname{dim} \mathcal{O}_{\Sigma, s}=K$, it follows that hd $\mathcal{O}_{\Sigma, s} \mathcal{O}_{C, x}=$ 0 . But this means that $\mathcal{O}_{C, x}$ is a free $\mathcal{O}_{\Sigma, s}$-module.

Proof of Theorem 1. Consider again the mapping $\pi: C \rightarrow \Sigma$ and let $s \in \Sigma$. For each $x \in C(s):=\pi^{-1}(s), \mathbb{C} \otimes_{\mathcal{O}_{\Sigma, s}} \mathcal{O}_{C, x}$ is a finite dimensional vector space over $\mathbb{C}$. Denote its dimension by $\nu(x)$. Define

$$
\nu(s)=\sum_{x \in C(s)} \nu(x) .
$$

By Theorem 2 and [D, $\S 5$, Theorem 1], $\nu(s)$ is a locally constant function of $s$. Now for a point $s=\left(s^{\prime}, \lambda\right)$ where $s^{\prime}$ is a regular value of $F, \nu(s)=\operatorname{ind}_{\mathbb{C}, 0} \omega$ by Proposition 3. On the other hand $\nu(0)=\operatorname{dim}_{\mathbb{C}} \mathcal{O}_{\mathbb{C}^{n}, 0} / I$.

\section{The real index as the signature of a family of quadratic forms}

Now we want to discuss the index of a real analytic 1-form $\omega$ on a real icis $(V, 0)=\left\{f_{1}=\ldots=f_{k}=0\right\} \subset\left(\mathbb{R}^{n}, 0\right)$ (with an algebraically isolated singular point at the origin). The 1-form $\omega$ on $V$ is the restriction of an analytic 1-form

$\sum_{i=1}^{n} A_{1} d x_{i}$ defined on $\mathbb{R}^{n}$ in a neighbourhood of the origin (we also denote this 1 -form by $\omega$ ). We consider the (analytic) functions $f_{1}, \ldots, f_{k}$ and the 1-form $\omega$ defined on a neighbourhood of the origin in $\mathbb{C}^{n}$ as well. Let $\delta$ be positive and small enough so that the functions $f_{1}, \ldots, f_{k}$ and the 1-form $\omega$ are defined on the ball $B_{\delta}$ of radius $\delta$ centred at the origin in $\mathbb{C}^{n}$ and for each positive $\delta^{\prime}<\delta$ the variety $V_{\mathbb{C}}=\left\{f_{1}=\ldots=f_{k}=0\right\} \subset\left(\mathbb{C}^{n}, 0\right)$ intersects the sphere $S_{\delta^{\prime}}$ of radius $\delta^{\prime}$ centred at the origin transversally. For $\varepsilon=\left(\varepsilon_{1}, \ldots, \varepsilon_{k}\right)$ small enough $(|\varepsilon| \ll \delta)$, let $V_{\mathbb{C}, \varepsilon}=\{f=\varepsilon\} \cap B_{\delta} \subset \mathbb{C}^{n}$, for $\varepsilon$ real, $V_{\varepsilon}=V_{\mathbb{C}, \varepsilon} \cap \mathbb{R}^{n}$. Let $\Sigma \subset\left(\mathbb{C}^{k}, 0\right)$ be the bifurcation diagram of the map $f$ (the set of $\varepsilon \in\left(\mathbb{C}^{k}, 0\right)$ critical for $f), \Sigma_{\mathbb{R}}=\Sigma \cap\left(\mathbb{R}^{k}, 0\right)$.

By Proposition 2, for real $\varepsilon$ outside of the bifurcation diagram (i.e., $\varepsilon \in$ $\mathbb{R}^{k} \backslash \Sigma_{\mathbb{R}}$ ), the (real) index ind $0 \omega$ differs from the sum of indices of zeros of the 1-form $\omega$ on the real smooth manifold $V_{\varepsilon}$ by $\left(\chi\left(V_{\varepsilon}\right)-1\right)$. By Proposition 3 the complex index ind $\mathbb{C}, 0_{0} \omega$ counts the number of zeros of the same 1-form on the complex manifold $V_{\mathbb{C}, \varepsilon}$. The Euler characteristic $\chi\left(V_{\varepsilon}\right)$ of the real level manifold $V_{\varepsilon}$ is different for different $\varepsilon \in \mathbb{R}^{k} \backslash \Sigma_{\mathbb{R}}$ (at least for $n-k$ even). It is constant on each component of the complement to the bifurcation diagram 
$\Sigma_{\mathbb{R}}$. Thus one cannot expect to get the (real) index $\operatorname{ind}_{0} \omega$ of the 1 -form $\omega$ as the signature of a nondegenerate quadratic form on a vector space of dimension $\operatorname{ind}_{\mathbb{C}, 0} \omega\left(=\operatorname{dim}_{\mathbb{C}} \mathcal{O}_{\mathbb{C}^{n}, 0} / I\right)$. Such a signature may be equal to ind $\operatorname{in}_{0} \omega+\left(\chi\left(V_{\varepsilon}\right)-1\right)$ and thus has to be different for different components of the complement to the bifurcation diagram. We want to obtain a somewhat finer description.

Theorem 3 There exists a family $Q_{\varepsilon}$ of quadratic forms on the space $\mathbb{C}^{L}$ of dimension $L=\operatorname{dim}_{\mathbb{C}} \mathcal{O}_{\mathbb{C}^{n}, 0} / I$ (i.e., a family of symmetric $L \times L$-matrices) defined for $\varepsilon \in \mathbb{C}^{k}$ from a neighbourhood of the origin and analytically dependent on $\varepsilon$ such that:

1) for $\varepsilon$ from the complement to the bifurcation diagram $\Sigma$, the quadratic form $Q_{\varepsilon}$ is nondegenerate;

2) for real $\varepsilon$, the quadratic form (i.e., the matrix) $Q_{\varepsilon}$ is real and, for real $\varepsilon$ outside of the bifurcation diagram (i.e., for $\varepsilon \in \mathbb{R}^{k} \backslash \Sigma_{\mathbb{R}}$ ), its signature is equal to

$$
\sum_{P \in V_{\varepsilon}} \operatorname{ind}_{P} \omega=\operatorname{ind}_{0} \omega+\left(\chi\left(V_{\varepsilon}\right)-1\right) .
$$

Proof. It is convenient to define the family $Q_{\varepsilon}$ for a larger space of parameters. Let $F:\left(\mathbb{C}^{n} \times \mathbb{C}^{M}, 0\right) \rightarrow\left(\mathbb{C}^{k} \times \mathbb{C}^{M}, 0\right)$ be a real (i.e., invariant with respect to the complex conjugation) versal deformation of the $f:\left(\mathbb{C}^{n}, 0\right) \rightarrow\left(\mathbb{C}^{k}, 0\right)$, $F\left(x, \varepsilon^{\prime}\right)=\left(f_{\varepsilon^{\prime}}(x), \varepsilon^{\prime}\right), f_{0}=f$. Here $\varepsilon_{k+1}, \ldots, \varepsilon_{k+M}$ are the coordinates on $\mathbb{C}^{M}$, $\varepsilon^{\prime}=\left(\varepsilon_{k+1}, \ldots, \varepsilon_{k+M}\right) \in \mathbb{C}^{M}$; let $\hat{\varepsilon}=\left(\varepsilon_{1}, \ldots, \varepsilon_{k}, \varepsilon_{k+1}, \ldots, \varepsilon_{k+M}\right) \in \mathbb{C}^{k+M}=$ $\mathbb{C}^{k} \times \mathbb{C}^{M}$. Let $\mathbb{C}_{\alpha}^{n}$ be the $n$-dimensional affine space with the coordinates $\alpha_{1}$, $\ldots, \alpha_{n}$, let $\varkappa=(\hat{\varepsilon}, \alpha)\left(\varkappa \in \mathbb{C}_{\varkappa}^{k+M+n}=\mathbb{C}^{k+M} \times \mathbb{C}_{\alpha}^{n}\right)$. Let us denote by the same letter, $F$, the trivial extension $\left(\mathbb{C}_{x}^{n} \times \mathbb{C}_{\varepsilon^{\prime}}^{M} \times \mathbb{C}_{\alpha}^{n}, 0\right) \rightarrow\left(\mathbb{C}_{\varepsilon}^{k} \times \mathbb{C}_{\varepsilon^{\prime}}^{M} \times \mathbb{C}_{\alpha}^{n}, 0\right)=$ $\left(\mathbb{C}_{\varkappa}^{k+M+n}, 0\right)$ of the chosen versal deformation: $F\left(x, \varepsilon^{\prime}, \alpha\right)=\left(f_{\varepsilon^{\prime}}(x), \varepsilon^{\prime}, \alpha\right)$. Let $\Omega$ be a 1 -form on $\left(\mathbb{C}_{x}^{n} \times \mathbb{C}_{\varepsilon^{\prime}}^{M} \times \mathbb{C}_{\alpha}^{n}, 0\right)$ defined by

$$
\Omega=\omega-\sum_{i=1}^{n} \alpha_{i} d x_{i}=\sum_{i=1}^{n}\left(A_{i}-\alpha_{i}\right) d x_{i} .
$$

Again by the same symbols, $\Sigma$ and $\Sigma_{\mathbb{R}}$, we shall denote the complex and the real bifurcation sets of the map $F$ (the real one being the intersection of the complex one with the real space $\left.\mathbb{R}^{k+M+n}\right), V_{\mathbb{C}, \varkappa}:=F^{-1}(\varkappa) \cap B_{\delta} \subset \mathbb{C}^{n+M+n}$, for $\varkappa$ real, $V_{\varkappa}:=V_{\mathbb{C}, \varkappa} \cap \mathbb{R}^{n+M+n}$ (in fact $V_{\mathbb{C}, \varkappa}$ is a subvariety of the $n$-dimensional affine space $\left.\mathbb{C}_{x}^{n} \times\left\{\left(\varepsilon^{\prime}, \alpha\right)\right\}\right)$.

For a point $P \in \mathbb{C}_{x}^{n} \times \mathbb{C}_{\varepsilon^{\prime}}^{M} \times \mathbb{C}_{\alpha}^{n}$ let

$$
\Delta=\Delta(P):=\left|\begin{array}{ccc}
\frac{\partial f_{\varepsilon^{\prime} 1}}{\partial x_{1}} & \cdots & \frac{\partial f_{\varepsilon^{\prime} 1}}{\partial x_{k}} \\
\vdots & \ddots & \vdots \\
\frac{\partial f_{\varepsilon^{\prime} k}}{\partial x_{1}} & \cdots & \frac{\partial f_{\varepsilon^{\prime} k}}{\partial x_{k}}
\end{array}\right|,
$$

where $f_{\varepsilon^{\prime} i}(i=1, \ldots, k)$ are the components of the map $f_{\varepsilon^{\prime}}: f_{\varepsilon^{\prime}}=\left(f_{\varepsilon^{\prime} 1}, \ldots, f_{\varepsilon^{\prime} k}\right)$. If $P \in V_{\mathbb{C}, \varkappa}$ and $\Delta(P) \neq 0$, the variables $x_{k+1}, \ldots, x_{n}$ are local coordinates 
on the $((n-k)$-dimensional $)$ variety $V_{\mathbb{C}, \varkappa}$ in a neighbourhood of the point $P$. For a generic $\varkappa \in\left(\mathbb{C}^{k+M+n}, 0\right) \backslash \Sigma$, for all zeros $P$ of the restriction of the 1 -form $\Omega$ to the level manifold $V_{\mathbb{C}, \varkappa}$, one has $\Delta(P) \neq 0$. Moreover, for a generic $\varkappa \in\left(\mathbb{C}^{k+M+n}, 0\right) \backslash \Sigma$ which does not possess this property (such $\varkappa$ 's form a subset of codimension 1), all zeros $P$ of the restriction of the 1-form $\Omega$ to the level manifold $V_{\mathbb{C}, \varkappa}$ are simple (i.e., nondegenerate).

For a local system of coordinates $y_{1}, \ldots, y_{m}$ on a manifold $M$ the Hessian $h=h_{\omega}$ of a 1 -form $\omega$ equal to $A_{1} d y_{1}+\ldots+A_{m} d y_{m}$ in these coordinates is defined as the determinant

$$
\left|\frac{\partial A_{i}}{\partial y_{j}}\right|_{i, j=1, \ldots, m} .
$$

(The Hessian is a function on the manifold $M$; it depends on the choice of the coordinates.)

For $i=k+1, \ldots, n$, let

$$
m_{i}:=\left|\begin{array}{cccc}
\frac{\partial f_{\varepsilon^{\prime} 1}}{\partial x_{1}} & \cdots & \frac{\partial f_{\varepsilon^{\prime} 1}}{\partial x_{k}} & \frac{\partial f_{\varepsilon^{\prime} 1}}{\partial x_{i}} \\
\vdots & \ddots & \vdots & \vdots \\
\frac{\partial f_{\varepsilon^{\prime} k}}{\partial x_{1}} & \cdots & \frac{\partial f_{\varepsilon^{\prime} k}}{\partial x_{k}} & \frac{\partial f_{\varepsilon^{\prime} k}}{\partial x_{i}} \\
A_{1}-\alpha_{1} & \cdots & A_{k}-\alpha_{k} & A_{i}-\alpha_{i}
\end{array}\right| .
$$

For a point $P \in V_{\mathbb{C}, \varkappa}$ with $\Delta(P) \neq 0$, let $h(P)$ be the Hessian of the 1-form $\Omega_{\mid V_{\mathbb{C}, \varkappa}}$ in the (local) coordinates $x_{k+1}, \ldots, x_{n}$.

Proposition 4 The Hessian $h$ of the 1-form $\Omega_{\mid V_{\mathbb{C}, \varkappa}}$ in the coordinates $x_{k+1}$, $\ldots, x_{n}$ is given by the formula:

$$
h=\frac{1}{\Delta^{2+(n-k)}}\left|\begin{array}{cccc}
\Delta & \frac{\partial \Delta}{\partial x_{1}} & \cdots & \frac{\partial \Delta}{\partial x_{n}} \\
0 & \frac{\partial f_{\varepsilon^{\prime} 1}}{\partial x_{1}} & \ldots & \frac{\partial f_{s^{\prime} 1}}{\partial x_{n}} \\
\vdots & \vdots & \ddots & \vdots \\
0 & \frac{\partial f_{s^{\prime} k}}{\partial x_{1}} & \ldots & \frac{\partial f_{\varepsilon^{\prime} k}}{\partial x_{n}} \\
m_{k+1} & \frac{\partial m_{k+1}}{\partial x_{1}} & \ldots & \frac{\partial m_{k+1}}{\partial x_{n}} \\
\vdots & \vdots & \ddots & \vdots \\
m_{n} & \frac{\partial m_{n}}{\partial x_{1}} & \cdots & \frac{\partial m_{n}}{\partial x_{n}}
\end{array}\right| .
$$

Lemma 2 In the coordinates $x_{k+1}, \ldots, x_{n}$ the 1 -form $\Omega_{\mid V_{\mathbb{C}, \varkappa}}$ can be expressed as

$$
\Omega_{\mid V_{\mathbb{C}, \varkappa}}=\frac{m_{k+1}}{\Delta} d x_{k+1}+\cdots+\frac{m_{n}}{\Delta} d x_{n} .
$$

Proof. One has

$$
0=d f_{\varepsilon^{\prime} 1}=\frac{\partial f_{\varepsilon^{\prime} 1}}{\partial x_{1}} d x_{1}+\cdots+\frac{\partial f_{\varepsilon^{\prime} 1}}{\partial x_{n}} d x_{n},
$$




$$
\begin{aligned}
& \vdots \quad \vdots \quad \vdots \\
& 0=d f_{\varepsilon^{\prime} k}=\frac{\partial f_{\varepsilon^{\prime} k}}{\partial x_{1}} d x_{1}+\cdots+\frac{\partial f_{\varepsilon^{\prime} k}}{\partial x_{n}} d x_{n} .
\end{aligned}
$$

By Cramer's rule

$$
\begin{aligned}
d x_{1} & =(-1)^{k} \frac{1}{\Delta}\left(\frac{\partial\left(f_{\varepsilon^{\prime} 1}, \ldots, f_{\varepsilon^{\prime} k}\right)}{\partial\left(x_{2}, \ldots, x_{k}, x_{k+1}\right)} d x_{k+1}+\cdots+\frac{\partial\left(f_{\varepsilon^{\prime} 1}, \ldots, f_{\varepsilon^{\prime} k}\right)}{\partial\left(x_{2}, \ldots, x_{k}, x_{n}\right)} d x_{n}\right), \\
\vdots & \vdots \quad \vdots \\
d x_{k} & =-\frac{1}{\Delta}\left(\frac{\partial\left(f_{\varepsilon^{\prime} 1}, \ldots, f_{\varepsilon^{\prime} k}\right)}{\partial\left(x_{1}, \ldots, x_{k-1}, x_{k+1}\right)} d x_{k+1}+\cdots+\frac{\partial\left(f_{\varepsilon^{\prime} 1}, \ldots, f_{\varepsilon^{\prime} k}\right)}{\partial\left(x_{1}, \ldots, x_{k-1}, x_{n}\right)} d x_{n}\right) .
\end{aligned}
$$

Substitution in the form $\Omega$ yields

$$
\Omega_{\mid V_{\mathbb{C}, \varkappa}}=\frac{m_{k+1}}{\Delta} d x_{k+1}+\cdots+\frac{m_{n}}{\Delta} d x_{n} .
$$

By some abuse of notation we shall denote partial derivatives in the coordinates $x_{k+1}, \ldots, x_{n}$ of functions on the manifold $V_{\mathbb{C}, \varkappa}$ by $\frac{d}{d x_{j}}(j=k+1, \ldots, n)$ (in order to distinguish them from partial derivatives of the corresponding functions on $\mathbb{C}^{n}$ ). Therefore the Hessian $h$ is the determinant of the matrix

$$
\left(\frac{d}{d x_{j}}\left(\frac{m_{i}}{\Delta}\right)\right)_{i, j=k+1, \ldots, n}
$$

For $1 \leq \ell \leq k$ and $k+1 \leq j \leq n$ we have

$$
\frac{d x_{\ell}}{d x_{j}}=(-1)^{k+\ell+1} \frac{1}{\Delta} \frac{\partial\left(f_{\varepsilon^{\prime} 1}, \ldots, f_{\varepsilon^{\prime} k}\right)}{\partial\left(x_{1}, \ldots, x_{\ell-1}, x_{\ell+1}, \ldots, x_{k}, x_{j}\right)} \text {. }
$$

Therefore we get

$$
\begin{aligned}
& \frac{d}{d x_{j}}\left(\frac{m_{i}}{\Delta}\right)=\frac{\partial}{\partial x_{j}}\left(\frac{m_{i}}{\Delta}\right)+\frac{\partial}{\partial x_{1}}\left(\frac{m_{i}}{\Delta}\right) \frac{d x_{1}}{d x_{j}}+\ldots+\frac{\partial}{\partial x_{k}}\left(\frac{m_{i}}{\Delta}\right) \frac{d x_{k}}{d x_{j}} \\
& =\frac{1}{\Delta^{3}}\left(\Delta \frac{\partial m_{i}}{\partial x_{j}} \Delta-m_{i} \frac{\partial \Delta}{\partial x_{j}} \Delta+(-1)^{k} \Delta \frac{\partial m_{i}}{\partial x_{1}} \frac{\partial\left(f_{\varepsilon^{\prime} 1}, \ldots, f_{\varepsilon^{\prime} k}\right)}{\partial\left(x_{2}, \ldots, x_{k}, x_{j}\right)}\right. \\
& -(-1)^{k} m_{i} \frac{\partial \Delta}{\partial x_{1}} \frac{\partial\left(f_{\varepsilon^{\prime} 1}, \ldots, f_{\varepsilon^{\prime} k}\right)}{\partial\left(x_{2}, \ldots, x_{k}, x_{j}\right)}+\ldots \\
& \left.-\Delta \frac{\partial m_{i}}{\partial x_{k}} \frac{\partial\left(f_{\varepsilon^{\prime} 1}, \ldots, f_{\varepsilon^{\prime} k}\right)}{\partial\left(x_{1}, \ldots, x_{k-1}, x_{j}\right)}+m_{i} \frac{\partial \Delta}{\partial x_{k}} \frac{\partial\left(f_{\varepsilon^{\prime} 1}, \ldots, f_{\varepsilon^{\prime} k}\right)}{\partial\left(x_{1}, \ldots, x_{k-1}, x_{j}\right)}\right) \\
& =\frac{1}{\Delta^{3}}\left|\begin{array}{ccccc}
\Delta & \frac{\partial \Delta}{\partial x_{1}} & \cdots & \frac{\partial \Delta}{\partial x_{k}} & \frac{\partial \Delta}{\partial x_{j}} \\
0 & \frac{\partial f_{\varepsilon^{\prime}}}{\partial x_{1}} & \cdots & \frac{\partial f_{\varepsilon^{\prime} 1}}{\partial x_{k}} & \frac{\partial f_{\varepsilon^{\prime} 1}}{\partial x_{j}} \\
\vdots & \vdots & \ddots & \vdots & \vdots \\
0 & \frac{\partial f_{\varepsilon^{\prime} k}}{\partial x_{1}} & \cdots & \frac{\partial f_{\varepsilon^{\prime} k}}{\partial x_{k}} & \frac{\partial f_{\varepsilon^{\prime} k}}{\partial x_{j}} \\
m_{i} & \frac{\partial m_{i}}{\partial x_{1}} & \cdots & \frac{\partial m_{i}}{\partial x_{k}} & \frac{\partial m_{i}}{\partial x_{j}}
\end{array}\right| .
\end{aligned}
$$


Proposition 1 now follows from the following two lemmas.

Lemma 3 Let $A$ be an invertible $l \times l$-matrix, $B$ an $l \times m$-matrix, $C$ an $m \times l$ matrix, and $D$ an $m \times m$-matrix. Then

$$
\operatorname{det}\left(\begin{array}{cc}
A & B \\
C & D
\end{array}\right)=\operatorname{det} A \cdot \operatorname{det}\left(D-C A^{-1} B\right) .
$$

Proof. Let $E_{m}$ denote the $m \times m$ identity matrix. Then we have

$$
\begin{aligned}
\operatorname{det}\left(\begin{array}{cc}
A & B \\
C & D
\end{array}\right) & =\operatorname{det} A \cdot \operatorname{det}\left(\begin{array}{cc}
A^{-1} & 0 \\
0 & E_{m}
\end{array}\right) \cdot \operatorname{det}\left(\begin{array}{cc}
A & B \\
C & D
\end{array}\right) \\
& =\operatorname{det} A \cdot \operatorname{det}\left(\begin{array}{cc}
E_{l} & A^{-1} B \\
C & D
\end{array}\right) \\
& =\operatorname{det} A \cdot \operatorname{det}\left(\begin{array}{cc}
E_{l} & A^{-1} B \\
C-C E_{l} & D-C A^{-1} B
\end{array}\right) \\
& =\operatorname{det} A \cdot \operatorname{det}\left(\begin{array}{cc}
E_{l} & A^{-1} B \\
0 & D-C A^{-1} B
\end{array}\right) \\
& =\operatorname{det} A \cdot \operatorname{det}\left(D-C A^{-1} B\right) .
\end{aligned}
$$

Lemma 4 Let $A$ be an invertible $l \times l$-matrix, $B$ an $l \times m$-matrix, $C$ an $m \times l$ matrix, and $D=\left(d_{i j}\right)$ an $m \times m$-matrix. Let $c^{i}$ denote the $i$-th row of $C$ and $b_{j}$ the $j$-th column of $B$. Let $H$ be the matrix

$$
\left(\left|\begin{array}{cc}
A & b_{j} \\
c^{i} & d_{i j}
\end{array}\right|\right)_{i, j=1, \ldots, m}
$$

Then

$$
\operatorname{det} H=(\operatorname{det} A)^{m-1}\left|\begin{array}{cc}
A & B \\
C & D
\end{array}\right| .
$$

Proof. By Lemma 3

$$
H=\left(\operatorname{det} A\left(d_{i j}-c^{i} A^{-1} b_{j}\right)\right)_{i, j=1, \ldots, m} .
$$

Another application of Lemma 3 yields

$$
\operatorname{det} H=(\operatorname{det} A)^{m} \operatorname{det}\left(D-C A^{-1} B\right)=(\operatorname{det} A)^{m-1}\left|\begin{array}{cc}
A & B \\
C & D
\end{array}\right| .
$$

For any $\varkappa \in \mathbb{C}^{k+M+n}$ and for any singular point $P$ of the 1 -form $\Omega$ on the (possibly singular) level variety $V_{\mathbb{C}, \varkappa}$ (i.e, for a singular point of $V_{\mathbb{C}, \varkappa}$ or for a 
zero of the 1-form on its smooth part), let $I_{P}$ be the ideal of $\mathcal{O}_{P, x}$ generated by the functions $f_{\varepsilon^{\prime} i}-\varepsilon_{i}(i=1, \ldots, k)$ and by the $(k+1) \times(k+1)$-minors of the matrix

$$
\left(\begin{array}{ccc}
\frac{\partial f_{\varepsilon^{\prime} 1}}{\partial x_{1}} & \cdots & \frac{\partial f_{\varepsilon^{\prime} 1}}{\partial x_{n}} \\
\vdots & \ddots & \vdots \\
\frac{\partial f_{\varepsilon^{\prime} k}}{\partial x_{1}} & \cdots & \frac{\partial f_{\varepsilon^{\prime} k}}{\partial x_{n}} \\
A_{1}-\alpha_{1} & \cdots & A_{n}-\alpha_{n}
\end{array}\right)
$$

Let $J_{P}=\mathcal{O}_{P, x} / I_{P}$. From the proof of Theorem 1 it follows that:

1) $\sum_{P \in V_{\mathbb{C}, \varkappa}} \operatorname{dim}_{\mathbb{C}} J_{P}=$ const $=\operatorname{dim}_{\mathbb{C}} J_{0}$;

2) if $\varphi_{1}, \ldots, \varphi_{L}$ are real elements of the ring $\mathcal{O}_{\mathbb{C}^{n}, 0}$ which are representatives of a basis of the factor-algebra $J_{0}$ (considered as analytic functions defined in a common neighbourhood of the origin in $\mathbb{C}^{n}$ ), then, for $\varkappa$ small enough, the multigerms of the functions $\varphi_{1}, \ldots, \varphi_{L}$ are representatives of a basis of the algebra $J_{\varkappa}=\oplus_{P \in V_{\mathrm{c}, \varkappa}} J_{P}$.

Thus the algebras $J_{\varkappa}$ form a trivial vector bundle over a neighbourhood of the origin in $\mathbb{C}^{k+M+n}$ and the choice of (real) representatives of the elements of the basis of the algebra $J_{0}$ fixes a trivialization of this bundle compatible with the real structure.

As it is usual in similar situations, a quadratic form $Q_{\varkappa}$ on the algebra $J_{\varkappa}$ is defined by the formula

$$
Q_{\varkappa}\left(\psi_{1}, \psi_{2}\right)=\ell_{\varkappa}\left(\psi_{1} \psi_{2}\right)
$$

where $\ell_{\varkappa}$ is a linear function on the vector space $J_{\varkappa}, \psi_{1} \psi_{2}$ means the product in the algebra $J_{\varkappa}$.

Let $\varkappa \in\left(\mathbb{C}^{k+M+n}, 0\right)$ be such that $\Delta(P) \neq 0$ for all singular points $P$ of the 1 form $\Omega_{\mid V_{\mathbb{C}, \varkappa}}$. In particular this means that $\varkappa$ is not contained in the discriminant $\Sigma$ of the deformation and the Hessian $h$ is defined in a neighbourhood of each singular point $P$. For a singular point $P$ of the 1 -form $\Omega_{\mid V_{\mathbb{C}, \varkappa}}$, let $\widetilde{h}_{P}:=h \cdot \Delta(P)^{2}$. For $\psi \in J_{\varkappa}$, define $\ell_{\varkappa}(\psi)$ as

$$
\sum_{P \in V_{\mathbb{C}, \varkappa}} \tilde{\ell}_{P}(\psi)
$$

where $\widetilde{\ell}_{P}(\psi)=0$ for $\psi$ from the summand $J_{P^{\prime}}$ for $P^{\prime} \neq P$,

$$
\tilde{\ell}_{P}(\psi)=\lim _{\beta \rightarrow 0} \sum \frac{\psi\left(a_{i}\right)}{\widetilde{h}_{P}\left(a_{i}\right)}
$$

for $\psi \in J_{P}, \beta=\beta_{k+1} d x_{k+1}+\ldots,+\beta_{n} d x_{n}$, the sum is over all simple (i.e., nondegenerate) zeros of the 1-form $\Omega-\beta$ which emerge from the (generally speaking, degenerate) zero $P$ for generic $\beta$. The expression under the limit sign is defined for a generic $\beta$ (i.e., for a generic $\beta$ the form $\Omega-\beta$ has only 
nondegenerate zeros). The facts that: it has a finite limit as $\beta$ tends to zero (as an element of $\mathbb{C}^{n-k}$ ), this limit depends analytically on $\varkappa$, the corresponding quadratic form is nondegenerate, it becomes real for $\varkappa$ real, and in the last case the signature of the corresponding real quadratic form is equal to

$$
\sum_{P \in V_{\varepsilon}} \operatorname{ind}_{P} \omega=\operatorname{ind}_{0} \omega+\left(\chi\left(V_{\varepsilon}\right)-1\right)
$$

- follow from the know properties of the similar objects for the smooth case (see, e.g., AGV], § 5).

At the moment the required linear functions $\ell_{\varkappa}$ (and thus the quadratic forms $Q_{\varkappa}$ ) are defined for $\varkappa$ outside of the discriminant $\Sigma$ and of the set $\Xi$ of those $\varkappa$ for which on $V_{\mathbb{C}, \varkappa}$ there exists a zero $P$ of the form $\Omega_{\mid V_{\mathbb{C}, \varkappa}}$ with $\Delta(P)=0$ (both $\Sigma$ and $\Xi$ are hypersurfaces in $\mathbb{C}^{k+M+n}$ ). We want to show that in fact this family of linear functions has an analytic extension to these two subsets as well. Moreover we should control the extension to the last subset so that the quadratic form $Q_{\varkappa}$ does not degenerate there.

To show that the constructed family of linear functions $\ell_{\varkappa}$ has an analytic extension to the set $\Xi \backslash \Sigma$ of those $\varkappa \in \mathbb{C}^{k+M+n} \backslash \Sigma$ for which there exists a zero $P$ of the form $\Omega_{\mid V_{\mathrm{C}, \varkappa}}$ with $\Delta(P)=0$, it is sufficient to prove that, for a nondegenerate zero $P$ of the form $\Omega_{\mid V_{\mathbb{C}, \varkappa}}$ with $\Delta(P)=0$, the linear function $\tilde{\ell}_{P^{\prime}}(\psi)$ has a finite limit different from zero as $P^{\prime}$ tends to $P$, where $P^{\prime}$ is a singular point of the 1 -form $\Omega_{\mid V_{\mathbb{C}, F\left(P^{\prime}\right)}}, F\left(P^{\prime}\right) \notin \Xi$. In this case $\operatorname{dim}_{\mathbb{C}} J_{P}=$ $\operatorname{dim}_{\mathbb{C}} J_{P^{\prime}}=1$, the vector spaces $J_{P}$ and $J_{P^{\prime}}$ are generated by one element $\varphi \equiv 1, \widetilde{\ell}_{P^{\prime}}(1)=\frac{1}{h_{P^{\prime}}\left(P^{\prime}\right)}$, and it is sufficient to show that $\widetilde{h}_{P^{\prime}}\left(P^{\prime}\right)$ has a finite nonzero limit as $P^{\prime} \rightarrow P$. Let $\sigma=\left\{j_{1}, \ldots, j_{n}\right\}$ be a permutation of the indices $1, \ldots, n$ such that the Jacobian $\Delta^{\prime}$ of the functions $f_{1}, \ldots, f_{k}$ with respect to the variables $x_{j_{1}}, \ldots, x_{j_{k}}$ at the point $P$ (and thus at all points $P^{\prime}$ close to $P$ ) is different from zero. In this case $x_{j_{k+1}}, \ldots, x_{j_{n}}$ are local coordinates on the manifold $V_{\mathbb{C}, \varkappa}$ at the point $P$ and thus at all points $P^{\prime}$ close to $P$ (on the corresponding level manifold). At a point $P^{\prime}$ close to $P$ and such that $\Delta\left(P^{\prime}\right) \neq 0$ as well (and thus where $x_{k+1}, \ldots, x_{n}$ are also local coordinates) the Jacobian of the coordinate change $x_{k+1}, \ldots, x_{n} \mapsto x_{j_{k+1}}, \ldots, x_{j_{n}}$ is equal to $\operatorname{sgn}(\sigma) \cdot \frac{\Delta^{\prime}\left(P^{\prime}\right)}{\Delta\left(P^{\prime}\right)}$. Thus the value of the Hessian of the restriction of the form $\Omega$ to the corresponding level manifold in the coordinates $x_{j_{k+1}}, \ldots, x_{j_{n}}$ at the point $P^{\prime}$ is equal to

$$
\frac{h\left(P^{\prime}\right) \Delta\left(P^{\prime}\right)^{2}}{\Delta^{\prime}\left(P^{\prime}\right)^{2}}
$$

and therefore differs from $\widetilde{h}\left(P^{\prime}\right)$ by a nonzero analytic factor. This finishes the proof in this case.

To show that the constructed family of linear functions $\ell_{\varkappa}$ has an analytic extension to the discriminant $\Sigma$ it is sufficient to prove the following. Let $P$ be a point at which the corresponding level variety $V_{\mathbb{C}, \varkappa}$ has a singularity of 
type $A_{1}$, the 1 -form $\Omega$ (as a 1 -form on $\mathbb{C}^{n+M+n}$ ) does not tend to zero, and the zero hyperplane of it is in general position with respect to the tangent cone of the variety $V_{\mathbb{C}, \varkappa}$ at the point $P$. In this case $\operatorname{dim}_{\mathbb{C}} J_{P}=2$. Let $\tilde{\varkappa}$ from a neighbourhood of $\varkappa$ be of the form $\left(\widetilde{\varepsilon}_{1}, \ldots, \widetilde{\varepsilon}_{k}, \varepsilon_{k+1}, \ldots, \varepsilon_{k+M}, \alpha_{1}, \ldots, \alpha_{n}\right)$ (i.e., $\tilde{\varkappa}$ differs from $\varkappa$ only by the first $k$ coordinates: the values of the functions $f_{1}$, $\left.\ldots, f_{k}\right)$ and such that $\tilde{\varkappa} \notin \Sigma$. The 1 -form $\Omega_{\mid V_{\mathbb{C}, \varkappa}}$ has two nondegenerate zeros $P_{1}=P_{1}(\widetilde{\varkappa})$ and $P_{2}=P_{2}(\widetilde{\varkappa})$. It is sufficient to show that the linear function $\tilde{\ell}_{P_{1}}+\tilde{\ell}_{P_{2}}$ has a finite limit as $\tilde{\varkappa} \rightarrow \varkappa$.

Without loss of generality one can suppose that $k=1, n \geq 2, P$ is the origin in $\mathbb{C}^{n}, f_{1}=x_{1}^{2}+\ldots+x_{n}^{2}, \varkappa=0, \omega(0)=d x_{1}$. The last equation means that $\omega=\left(1+C_{1}\right) d x_{1}+\ldots+C_{n} d x_{n}$, where $C_{i} \in \mathfrak{m}$, i.e., $C_{i}(0)=0$. Let $\tilde{\varkappa}=\varepsilon^{2}$. As a basis of the algebra $J_{P}$ (as a vector space) and thus of the algebra $J_{P_{1}} \oplus J_{P_{2}}$ one can take $\varphi_{1} \equiv 1$ and $\varphi_{2}=x_{1}$. For the coordinates of the points $P_{1}$ and $P_{2}$ one has $x_{1}= \pm \varepsilon+o(\varepsilon), x_{i}=o(\varepsilon)$ for $i \geq 2$. Here and further on all series are power series in $\varepsilon$ and thus, for example, $o(\varepsilon)$ means $a_{2} \varepsilon^{2}+$ terms of higher degree. From Proposition 1 it follows that $h\left(P_{i}\right)=$ $(-1)^{n-1}( \pm \varepsilon)^{1-n}+$ terms of higher degree and thus $\widetilde{h}\left(P_{i}\right)=(-1)^{n-1}( \pm \varepsilon)^{3-n}+$ $\ldots$ Therefore $\tilde{\ell}_{P_{1}}(1)+\tilde{\ell}_{P_{2}}(1)=(-1)^{n-1}\left(\varepsilon^{n-3}+(-\varepsilon)^{n-3}\right)+\ldots, \widetilde{\ell}_{P_{1}}\left(x_{1}\right)+$ $\tilde{\ell}_{P_{2}}\left(x_{1}\right)=(-1)^{n-1}\left(\varepsilon^{n-2}+(-\varepsilon)^{n-2}\right)+\ldots$ Both expressions have finite limits as $\varepsilon$ tends to zero (for $n=2$, the terms $\varepsilon^{-1}$ and $(-\varepsilon)^{-1}$ sum up to zero).

Remarks. 1) To prove Theorem 3 actually it was not necessary to make precise computations for the $A_{1}$ case. It is clear that the components of the linear function $\ell_{\varkappa}$ (its values on the basis elements) can have only power asymptotics when $\varepsilon \rightarrow 0$. Thus multiplying the constructed functions by a suitably high power of the equation of the discriminant we obtain the required family.

2) The calculations above for the $A_{1}$-singularity show that for $n-k=1$, i.e., for curves, the constructed family of quadratic forms does not degenerate anywhere (including the discriminant) and, in particular, the quadratic form $Q_{0}$ (defined on the algebra $J_{0}$ ) is nondegenerate and its signature is equal to the same expression (2).

3) In [Sz2] there was proved a somewhat similar statement which in our terms can be considered as a particular case for the 1-form $\omega$ being the differential $d f_{k+1}$ of a function. However the family of quadratic forms defined there on the target space of the map $f: \mathbb{C}^{n} \rightarrow \mathbb{C}^{k}$ could degenerate at points $\varepsilon \in \mathbb{C}^{k}$ for which there exists a zero $P$ of the 1 -form $\omega$ on the level manifold $V_{\mathbb{C}, \varepsilon}$ with $\Delta(P)=0$ (and maybe somewhere else). (By the way, in some particular cases all points $\varepsilon$ from the target $\mathbb{C}^{k}$ could possess this property.) Thus one can say that our result gives a certain improvement of the one from Szz2] for $\omega=d f_{k+1}$ as well. 


\section{References}

[ASV] Aguilar, M. A., Seade, J. A., Verjovsky, A.: Indices of vector fields and topological invariants of real analytic singularities. J. reine angew. Math. 504, 159-176 (1998).

[A] Arnold, V. I.: Indices of singular points of 1-forms on a manifold with boundary, convolution of invariants of reflection groups, and singular projections of smooth surfaces. Uspekhi Mat Nauk 34:2, 3-38 (1979) (Engl. translation in Russian Math. Surveys 34:2, 1-42 (1979)).

[AGV] Arnold, V. I., Gusein-Zade, S. M., Varchenko, A. N.: Singularities of Differentiable Maps, Vol. I, Birkhäuser, Boston Basel Berlin, 1985.

[BG] Bonatti, Ch., Gómez-Mont, X.: The index of holomorphic vector fields on singular varieties I. Astérisque 222, 9-35 (1994).

[BR] Buchsbaum, D. A., Rim, D. S.: A generalized Koszul complex. II. Depth and multiplicity. Trans. Am. Math. Soc. 111, 197-224 (1964).

[D] Douady, A.: Flatness and privilege. L'Enseignement Math. 14, 47-74 (1968).

[EGZ] Ebeling, W., Gusein-Zade, S. M.: On the index of a vector field at an isolated singularity. In: The Arnoldfest: Proceedings of a Conference in Honour of V. I. Arnold for his Sixtieth Birthday (E. Bierstone, B. Khesin, A. Khovanskii, J. Marsden, eds.), Fields Institute Communications, Vol. 24, Am. Math. Soc., Providence 1999, pp. 141-152.

[EL] Eisenbud, D., Levine, H. : An algebraic formula for the degree of a $C^{\infty}$ map germ. Ann. Math. 106, 19-38 (1977).

[GM] Gómez-Mont, X.: An algebraic formula for the index of a vector field on a hypersurface with an isolated singularity. J. Alg. Geom. 7, 731752 (1998).

[GMM1] Gómez-Mont, X., Mardešić, P.: The index of a vector field tangent to a hypersurface and the signature of the relative Jacobian determinant. Ann. Inst. Fourier 47, 1523-1539 (1997).

[GMM2] Gómez-Mont, X., Mardešić, P.: The index of a vector field tangent to an odd-dimensional hypersurface and the signature of the relative Hessian. Funkt. Anal. i Prilozhen. 33, 1-13 (1999) (Engl. translation in Funct. Anal. Appl. 33, 1-10 (1999).

[GSV] Gómez-Mont, X., Seade, J., Verjovsky, A.: The index of a holomorphic flow with an isolated singularity. Math. Ann. 291, 737-751 (1991). 
[G] Greuel, G. M.: Der Gaus̈-Manin-Zusammenhang isolierter Singularitäten von vollständigen Durchschnitten. Math. Ann. 214, 235-266 (1975).

[H] Husemoller, D.: Fibre bundles. Second Edition. Graduate Texts in Math. 20, Springer-Verlag, New York Heidelberg Berlin 1975.

[Kh] Khimshiashvili, G. N.: On the local degree of a smooth map. Comm. Acad. Sci. Georgian SSR. 85, no. 2, 309-311 (1977) (in Russian).

[L] Looijenga, E. J. N.: Isolated singular points on complete intersections. London Math. Soc. Lecture Note Series 77, Cambridge University Press, Cambridge 1984.

[Ma] Matsumura, H.: Commutative algebra. Second edition. Benjamin/Cummings, Reading, Massachusetts 1980.

[Mi] Milnor, J.: Singular points of complex hypersurfaces. Annals of Math. Studies 61, Princeton University Press, Princeton 1968.

[SS] Seade, J. A., Suwa, T.: A residue formula for the index of a holomorphic flow. Math. Ann. 304, 621-634 (1996).

[Sz1] Szafraniec, Z.: A formula for the Euler characteristic of a real algebraic manifold. manuscripta math. 85, 345-360 (1994).

[Sz2] Szafraniec, Z.: On topological invariants of real analytic singularities. Math. Proc. Camb. Phil. Soc. 130, 13-24 (2001).

Institut für Mathematik, Universität Hannover, Postfach 6009, D-30060 Hannover, Germany

E-mail: ebeling@math.uni-hannover.de

Department of Mathematics and Mechanics,

Moscow State University

Moscow, 119899, Russia

E-mail: sabir@mccme.ru 